\title{
INTRA-ARTERIAL OXYGEN IN THE MANAGEMENT OF ISCHAEMIC GANGRENE IN THE ELDERLY
}

\author{
By D. J. MarTin, M.B., B.S. (Lond.), F.R.C.S.(Ed.) \\ Orthopaedic Surgeon, West Suffolk General Hospital, Bury St. Edmunds
}

The lot of the old person who comes to amputation is a sad one. Many have been living lives of personal independence, even if it was a limited one, but after amputation they need much more help and those with whom they lived often find themselves unable to give that extra help. Though some may manage after the loss of one limb, they become very much more helpless and dependent following a second major amputation. An increasing number of people are having to face this problem with the increasing longevity of our population. It has, therefore, become a matter of importance to find ways of lessening this damage.

It is important, first of all, to take steps to lessen the risks of those suffering from obliterative vascular disease. The aching of legs and feet may be found to be the result of defective circulation rather than arthritis, and this is a possibility that should be borne in mind.

If feet can be protected from minor trauma resulting from ill-fitting shoes, carelessly cut corns or nails and neglected cracks between toes, the circulation will meet ordinary demands when it would prove inadequate for any inflammatory process. Feet should be kept warm so as to prevent damage following peripheral vasoconstriction. On the other hand, hot-water bottles should be used with caution to avoid making the feet sweat and increasing metabolism. When washing the feet care should be taken to dry them thoroughly, especially between the toes, and the use of a dusting powder will facilitate this. There is much evidence to show that tobacco will increase vasospasm (Lynn and Burt, 1949). Alcohol, on the other hand, may be of real help in the elderly, for, though its vasodilator properties may not be limited, it is very useful as a sedative.

The large number of preparations now on the market to produce peripheral vasodilatation is evidence of the amount of thought that this subject has evoked. To give these preparations by mouth may be almost valueless, as the part of the body most in need of them will be the very part which will gain the smallest supply through its defective circulation. Their use can prove to be even harm- ful, through the general lowering of blood pressure To give them intra-arterially is more logical and has given some good results (Edwards, I952). It is a method that has been used in our clinic over the past three years, but the results have not been good enough in old people with peripheral vascular disease.

A considerable amount of work has been done recently on the value of intra-arterial injections of gases. Lemaire was amongst the first to use it for diagnosis and treatment of arterial diseases (Lemaire, 1950). Of these, oxygen has been found to be safe and a good vasodilator. Compared with drugs of the acetylcholine group, it has been shown to be as good a dilator and to give rise to more lasting effects (Marshall and Whelan, 1956). This has been applied to the clinical field, especially by German workers (Judmaier, I95I). It is, of course, impossible to avoid amputation once gangrene has developed, but it does seem that with treatment of this sort it is possible to carry out local amputations, so that much helplessness is avoided or, at any rate, it is possible to amputate at a lower level than was thought to be safe formerly. The lower the level, the less the operative risk, and the less the damage to morale.

It is probable that the action of the gas is a mechanical one, for it has been shown that the same gas in solution will not produce the same results as when insufflated in a gaseous form (Duff, Greenfield and Whelan, 1953). The immediate effect is to cause blanching of the skin due to arteriospasm, but in the course of a few minutes areas of redness appear in the skin and these gradually become more diffuse until a blush is seen throughout the limb. The dilatation lasts from about half an hour up to about 48 hours.

In trying to assess the amount of benefit available from this form of treatment, plans have been made to devise objective measurements to show an increased circulation. As little reliance as possible has been placed on the patient's own report of improvement and relief from pain. In addition to an ordinary physical examination, before treatment is begun an oscillograph tracing is taken as an 
indication of the amount of blood flowing into each part of the limb. This can be repeated at the end of the course of treatment and may sometimes give enough information to avoid the an arteriogram in demonstrating the position of an arterial block. By the use of an electric thermometer the differences in skin temperatures in different parts of a limb can be measured, and if at the end of the treatment it is found that the differences have become less it would be reasonable to deduce an increased blood supply (von Ratschow, 1954). Colour photographs are also a useful way of recording progress. In those patients whose chief complaint is intermittent claudication the colour does not often vary much from normal, and in these it is possible to get some idea of improvement by the ability to walk increasing distances on a level roadway marked out in 10-yd. strips. This by itself, however, is a notoriously fallacious way of assessing improvement. Having taken every precaution, in the end it is impossible to avoid the general sense of well-being experienced by a patient who finds himself treated enthusiastically for a long-standing and progressive condition.

For clinical use of oxygen it is necessary to have a simple apparatus, which can be taken from ward to ward and used in an out-patient department readily. Such an apparatus has been devised by Möller, modifying that in use at Innsbruck. This apparatus consists of a small oxygen cylinder from which the gas flows through a manometer that will allow the gas to enter the circulation at just about systolic pressure. This is connected to a second manometer, which shows the actual pressure in the artery. This makes a certain demonstration that the needle has been introduced into an artery and not a vein, as it shows the variation from systolic to diastolic pressure. From here the gas is led through a tube of water, through which the bubbles can easily be seen and the rate of flow determined. Then it is led to a cylinder with a piston that allows 20 c.c. of gas to pass with each excursion of the piston. A long plastic tube leads the gas to the needle introduced into the artery. There is a drip chamber immediately before the needle, into which the blood can be seen to flow back as the needle is introduced into the vessel.

The most convenient position for the patient is to have him on a firm couch, with a firm cushion under his buttocks and his hip hyper-extended and externally rotated. There is apparently no risk of an air embolism occurring during the injections into the femoral artery and very little into the brachial. One case has been reported of transient unconsciousness following injection into the brachial artery, and one death following an injection into the subclavian artery (Lemaire, 1948).
The theoretical risk of producing a thrombosis does not seem to have occurred in practice. There would seem to be a risk of producing an aneurysm $\mathbb{\AA}$ at the site of repeated injections in the elderly $c$. people with calcified arteries, and so it is wise to $\underset{\vec{D}}{\vec{D}}$ spread the point of puncture as much as possible. Some leaking may occur, leading to a haematoma. To prevent this the patient is instructed to keep a $\frac{\overline{5}}{\bar{N}}$ swab firmly pressed on the site of the injection for $\frac{\vec{\sigma}}{\sigma}$ a few minutes, and this serves the double purpose $\triangleright$ of lessening the risk of haematoma and in keeping $\%$ him lying flat for a few minutes after introducing $\overrightarrow{0}$ gas into his circulation.

Amounts of 60 to 80 c.c. are introduced at each insufflation and a course of eight to twelve injections are given bi-weekly. These beneficial results pass off in about six months and patients are instructed to report again in that time, or before, if symptoms recur.

Results are difficult to assess, as it is reasonable to expect improvement in any case through general की care and local treatment given. Our cases are judged on the following criteria:

r. Improvement in circulation, demonstrated by oscillograph and skin temperature measurements.

2. Ability to walk longer distances without pain,

3. Sleep less disturbed by resting pain.

4. Patient's report of subjective improvement.

5. Pulses palpable that were not formerly paF pable.

6. Improved colour.

7. Increased rate of healing of gangrenous lesions.

In addition to cases of arteriosclerotic ischaemia and diabetic gangrene, this form of treatment is also of value in treating Buerger's disease, Raynaud's disease, erythrocyanosis cruris puellarum, frostbite, chilblains, circulatory defects complicating anterior poliomyelitis, and sometimes varicose ulcers.

Contra-indications are acute thrombotic episodes, auricular fibrillation and advanced and $\risingdotseq$ extensive gangrene, as the oxygen bubbles may 0 occlude the few remaining vessels.

\section{BIBLIOGRAPHY}

DUFF, F., GREENFIELD, A.D.M., and WHELAN, R.F. (1953), N Lancet, ii, 230.

EDWARDS, J. W. L. (1952), Brit. Med. f., 2, 808.

JUDMAIER, P. (1951), Munch. med. Wschr., 93, 1437.

LEMAIRE, A. (1950), Wien. klin. Wschr., 62, 1.

LEMAIRE, A. (1948), Bull. Acad. Nat. de Med., 132, 384. LYNN, A. B., and BURT, C. C. (1949), Brit. med. F., if, 808. MARSHALL, R. J., and WHELAN, R. F. (1956), Ibid., II, 344. VON RATSCHOW, M. (1954), Med. Klin., 49, 691. 